\title{
DETERMINANTES DAS ESCOLHAS INOVATIVAS E DE APRENDIZAGEM DOS FRUTICULTORES CEARENSES ${ }^{1}$
}

\author{
Eliane Pinheiro de Sousa ${ }^{2}$ \\ Wellington Ribeiro Justo ${ }^{3}$ \\ Antônio Carvalho Campos ${ }^{4}$
}

\begin{abstract}
Resumo: Este estudo objetiva identificar as variáveis que influenciam as escolhas inovativas e de aprendizagem dos fruticultores localizados nas seis regiões cearenses irrigadas produtoras de frutas. Para tal, empregou-se o modelo de regressão quantílica, que permite captar os efeitos desses determinantes ao longo de pontos distintos da distribuição condicional dos índices de inovação tecnológica e de aprendizagem e não somente na média. Foram utilizados dados primários coletados diretamente com 150 fruticultores. Os resultados mostraram que a escolaridade é uma variável relevante na explicação das diferenças de inovações tecnológicas e de fontes de informação para promoção do aprendizado da atividade frutícola em todos os quantis estimados. A assistência técnica também apresentou efeito positivo sobre as escolhas inovativas ao longo da distribuição. Ademais, as variáveis dummies regionais Baixo Acaraú e Ibiapaba indicaram diferencial de inovação tecnológica e de aprendizagem em relação à variável Metropolitana apenas nos quantis inferiores.
\end{abstract}

Palavras-chave: inovação tecnológica; aprendizagem; regressão quantílica.

Recebido em: 17/10/11. Aceito em: 26/3/2012.

2 Doutora em Economia Aplicada pela Universidade Federal de Viçosa (UFV), Pesquisadora da Fundação Cearense de Apoio ao Desenvolvimento Científico e Tecnológico (FUNCAP) e Professora Adjunta do Departamento de Economia da Universidade Regional do Cariri (URCA).E-mail: pinheiroeliane@hotmail.com

3 Doutor em Economia - PIMES pela Universidade Federal de Pernambuco (UFPE) e Professor Adjunto do Departamento de Economia da Universidade Regional do Cariri (URCA). E-mail: justowr@yahoo.com.br

4 Ph.D. in Agricultural Economics e Professor Titular do Programa de Pós-Graduação em Economia Aplicada da Universidade Federal de Viçosa (UFV). E-mail: accampos@ufv.br 


\begin{abstract}
This study aims to identify the variables that influence the innovative and learning choices of the fruit farmers located in the six regions of the State of Ceará dedicated to fruit farming. In order to do that, it was employed the quantile regression model, that enables to observe the effects of these determinants along distinct points of the conditional distribution of the technological innovation and learning indexes and not only along the average. It was used primary data collected directly from 150 fruit farmers. Results showed that schooling level is a relevant variable in explaining differences in technological innovations and information sources for the promotion of learning of the fruit farming activity in all estimated amounts. Technical assistance also displayed positive effect on the innovative choices throughout the distribution. In addition, the regional dummies variables Baixo Acaraú and Ibiapaba indicated differential of technological innovation and learning in relation to the variable Metropolitan only on the inferior amounts.
\end{abstract}

Keywords: technological innovation; learning; quantile regression.

\title{
1. Introdução
}

O setor agrícola assume papel importante para o estado do Ceará, não somente pela produção de alimentos, como também pela geração de emprego e renda, contribuindo para a redução da migração rural-urbana. Dentro do setor agrícola, a fruticultura irrigada tem-se destacado como atividade dinâmica, produzindo alimentos de maior valor agregado (SILVA et al., 2004).

Conforme dados divulgados no jornal Diário do Nordeste (2009), a fruticultura irrigada cearense consolida-se como um dos principais segmentos exportadores do Estado, sendo responsável por cerca de 21 mil empregos diretos. O Ceará vem conquistando posição no mercado internacional de frutas tropicais, haja vista ter ocupado a $3^{\mathrm{a}}$ colocação no ranking nacional de exportações de frutas, atrás apenas de Pernambuco e da Bahia.

Não obstante esses resultados, Souza e Ponciano (2007) destacam que a fruticultura necessita de um conjunto de habilidades e cuidados que precisam ser desenvolvidos. Segundo esses autores, ao citar BNB (2006), 
a fruticultura caracteriza-se por ser muito exigente em termos tecnológicos, desde o cultivo até a pós-colheita. Aspectos relacionados à deficiência no controle fitossanitário e pouca pesquisa quanto a variedades, tecnologias de produção e tratamento pós-colheita comprometem sobremaneira a produtividade de frutas. Ademais, o baixo nível de conhecimento dos fruticultores também limita a competitividade.

Nesse contexto, torna-se relevante a realização de estudos que levem em consideração essas questões concernentes aos condicionantes dos níveis tecnológicos e de aprendizagem por meio de fontes de informações que propiciem aos produtores conhecimento do setor frutícola. $\mathrm{Na}$ literatura econômica, tem-se comumente buscado identificar as variáveis que condicionam a probabilidade de adoção do nível tecnológico, considerando o índice tecnológico uma variável dependente na forma dicotômica, e estimando tais determinantes via modelo logit ou probit. Estudos deste tipo foram desenvolvidos, por exemplo, por Oliveira (2003) e Matos (2005), que buscaram identificar os efeitos de variáveis socioeconômicas sobre o nível tecnológico da bananicultura e da apicultura em municípios cearenses, respectivamente.

A inovação deste estudo em relação aos realizados é que se busca verificar os efeitos das variáveis socioeconômicas sobre o índice de inovação tecnológica por meio do modelo de regressão quantílica, permitindo captar os efeitos desses determinantes ao longo de pontos distintos da distribuição condicional desse índice. Além disso, busca-se adicionalmente identificar as variáveis que influenciam o índice de aprendizagem dos fruticultores.

Portanto, pretende-se neste artigo identificar os determinantes das escolhas inovativas e de aprendizagem dos produtores de frutas localizados em seis regiões de fruticultura irrigada.

Além dessa seção introdutória, o artigo conta com mais três seções, sendo que a segunda aborda os procedimentos metodológicos do estudo. Em seguida, os resultados são mostrados e discutidos e, por fim, apresentadas as conclusões do estudo. 


\section{Procedimentos metodológicos}

\subsection{Método de análise}

Para avaliar o impacto da inovação tecnológica e da aprendizagem dos fruticultores em diferentes pontos da distribuição condicional, empregouse o método de regressão quantílica, elaborado a princípio por Koenker e Bassett (1978).

De acordo com Maciel et al. (2001), ao citar Buchinsky (1997), a regressão quantílica apresenta características interessantes como poder ser empregada para caracterizar toda a distribuição condicional de uma variável resposta dado um conjunto de regressores; possuir a forma de programação linear, o que facilita a estimação dos parâmetros; o vetor de coeficientes estimados não ser sensível a observações discrepantes na variável dependente; os estimadores da regressão quantílica poderem ser mais eficientes que os estimadores dos mínimos quadrados ordinários quando os erros não apresentam distribuição normal. Ademais, permite que soluções diferenciadas para quantis distintos possam ser interpretadas como diferenças na resposta da variável dependente às alterações nos regressores em diversos pontos da distribuição condicional da variável dependente.

Conforme Koenker e Basset (1978), a regressão quantílica $\theta$ pode ser representada a partir da solução de:

$$
\min _{\beta} \frac{1}{n_{i: y_{i}>x_{i} \beta}} \sum_{i} \theta\left|y_{i}-x_{i}^{\prime} \beta_{\theta}\right|+\sum_{: y_{i} \leq x_{i}^{\prime} \beta}(1-\theta)\left|y_{i}-x_{i}^{\prime} \beta_{\theta}\right|=\min _{\beta} \frac{1}{n} \sum_{i=1}^{n} \rho_{\theta}\left(\mu_{\theta_{i}}\right)
$$

em que $\rho_{\theta}$ é a função check definida por

$$
\rho_{\theta}\left(u_{\theta_{i}}\right)=\left\{\begin{array}{c}
\theta u_{\theta_{i}}, \quad \mathbf{u}_{\theta_{i}} \geq 0 \\
(\theta-1) u_{\theta_{i}}, \mathbf{u}_{\theta_{i}}<0
\end{array}\right.
$$


Segundo Justo (2008), os coeficientes da regressão quantílica podem ser interpretados por meio da derivada parcial do quantil condicional em relação a um regressor particular, ou seja, tais coeficientes podem ser interpretados como uma variação marginal no $\theta_{t h}$ quantil condicional ocasionada por uma mudança no regressor.

Considerando que as variáveis explicativas não influenciam os diferentes níveis de inovação tecnológica e de aprendizagem da mesma forma, foram estimadas regressões para os quantis 0,$25 ; 0,50$ e 0,75 , para verificar os efeitos dos fatores determinantes da inovação tecnológica e da aprendizagem ao longo da distribuição, em que o $\theta_{t h}$ quantil condicional do nível de inovação tecnológica e de aprendizagem pode ser expresso por:

$Q_{\theta}\left(y_{i} \mid x_{1}, x_{2}, x_{3}, x_{4}, x_{5}, x_{6}, x_{7}, x_{8}, x_{9}\right)=\beta_{0}+\sum_{j=1}^{9} \beta_{j} x_{j}, \theta \in[0,1]$ e $j=1,2, \ldots, 9$.

em que $y_{i}$ corresponde aos índices de inovação tecnológica (IIT) e de aprendizagem (IA); $x_{1}$ refere-se à prática de atividades cooperativas; $x_{2}$, à assistência técnica; $x_{3}$, à escolaridade; $x_{4}$, ao crédito agrícola; $x_{5}$, ao Baixo Acaraú; $x_{6}$, ao Baixo Jaguaribe; $x_{7}$, ao Cariri; $x_{8}$, ao Centro-Sul; e $x_{9}$, a Ibiapaba.

A operacionalização desse modelo, descrito em (3), foi feita por meio do software STATA 11. 


\subsection{Variáveis consideradas}

Como o interesse deste estudo se destina à identificação das variáveis que influenciam as escolhas inovativas e de aprendizagem dos produtores, então foram consideradas as variáveis relativas ao índice de inovação tecnológico (IIT) e de aprendizagem (IA) como regressores do modelo de regressão quantílica. Seguindo as definições sugeridas por Sousa e Campos (2010), o índice que mede a atividade inovativa dos fruticultores pode ser expresso por:

IIT $=\frac{\left(\sum \text { Rotineira }{ }^{*} 1,0+\sum \text { Ocasional }{ }^{*} 0,5+\sum \text { Não _ desenvolve }{ }^{*} 0\right)}{N}$

em que $\mathrm{N}$ corresponde ao número de eventos considerados. Para determinação desse índice, foram levados em conta os seguintes eventos: (i) Aquisição de máquinas e equipamentos que geraram melhorias tecnológicas; (ii) Programa de treinamento destinado à introdução de produtos e/ou processos; e (iii) Novas formas de comercialização e distribuição de produtos novos no mercado.

Assim, conforme se observa pela expressão (4), esse índice é ponderado pela frequência de inovações empregadas pelo produtor de fruta entrevistado, de forma que foi atribuído peso um para as inovações adotadas pelo produtor de forma rotineira; peso 0,5 , para o produtor que desenvolveu inovações ocasionalmente; e peso zero, para o que não realizou inovações em 2008.

Por sua vez, seguindo o estudo de Sousa e Campos (2010), o índice de aprendizagem (IA) capta a importância dada pelos fruticultores entrevistados a um dado conjunto de eventos, sendo determinado por:

$I A=\frac{\left(\sum \text { Alta_imp. }{ }^{*} 1,0+\sum \text { Média_imp. }{ }^{*} 0,6+\sum \text { Baixa_imp. }{ }^{*} 0,3+\sum \text { Irrelevante }{ }^{*} 0\right)}{N}$ 
Os eventos que compõem esse índice se referem às fontes de informações adotadas para o aprendizado da atividade frutícola que são os seguintes: (i) Aprendizagem interna; (ii) Aprendizagem com outros agentes produtivos; (iii) Universidades e/ou institutos de pesquisa; (iv) Conferências, seminários e cursos; (v) Participação em feiras; e (vi) Informações de rede baseadas na internet.

O estudo contou também com as seguintes variáveis explicativas a serem incluídas na estimação dos modelos: $x_{1}$ refere-se à prática de atividades cooperativas; $x_{2}$, à assistência técnica; $x_{3}$, à escolaridade; $x_{4}$, ao crédito agrícola; $x_{5}$, ao Baixo Acaraú; $x_{6}$, ao Baixo Jaguaribe; $x_{7}$, ao Cariri; $x_{8}$, ao Centro-Sul; e $x_{9}$, a Ibiapaba.

Com relação a essas variáveis, é importante ressaltar que cooperativismo, assistência técnica e crédito correspondem às variáveis dummies, em que se assume valor um, se o produtor tiver acesso a essas atividades, e zero, caso contrário. No caso da variável escolaridade, considerou-se como função dos anos de estudo.

O modelo contém também as dummies regionais para verificar se existe alguma diferença regional quanto às escolhas inovativas e de aprendizagem. A variável dummy regional considerada referência para as demais regiões, e, portanto omitida neste estudo, foi a região Metropolitana. Essa escolha foi baseada no critério dos maiores valores médios dos índices de inovação tecnológico (IIT) e de aprendizagem (IA). Portanto, todos os coeficientes estimados para as dummies regionais devem ser analisados como diferenciais relativos aos estimados pela região Metropolitana. Nesse contexto, as variáveis Baixo Acaraú, Baixo Jaguaribe, Cariri, Centro-Sul e Ibiapaba assumirão valor igual à unidade se o fruticultor pertencer à região em análise e zero, caso contrário. 


\section{3. Área de estudo, fonte dos dados e amostragem}

De acordo com dados do IBGE/SDA/Instituto Agropolos, referentes a 2007, fornecidos pelo Diretor de Agronegócios da Agência de Desenvolvimento Econômico do Ceará (ADECE), 82,3\% do plantio irrigado cearense de frutas se concentra nas regiões do Baixo Acaraú, Baixo Jaguaribe, Cariri, Centro-Sul, Ibiapaba e Metropolitana. Dada a expressividade da produção de frutas nessas áreas irrigadas, elas foram escolhidas para este estudo.

A área de fruticultura irrigada do Baixo Acaraú foi representada pelo Perímetro Irrigado Baixo Acaraú do Departamento Nacional de Obras contra as Secas (DNOCS), que abrange áreas dos municípios de Acaraú, Bela Cruz e Marco. Para representar a área de fruticultura irrigada do Baixo Jaguaribe, foram considerados os Perímetros Irrigados Tabuleiros de Russas e Jaguaribe Apodi do DNOCS, localizados nos municípios de Limoeiro do Norte, Russas e Morada Nova. No caso da área irrigada do Cariri, tomou-se como referência a produção de frutas proveniente do município de Mauriti e no Centro-Sul, considerou-se o município de Iguatu como representativo desta região. No tocante à área de fruticultura irrigada da Ibiapaba, foram selecionados os municípios de Ubajara e Tianguá para representar esta região, enquanto a fruticultura irrigada da região Metropolitana foi baseada no município de Maranguape.

Os dados empregados neste estudo foram obtidos mediante pesquisa de campo realizada durante os meses de janeiro e fevereiro de 2009 diretamente com os produtores de frutas dessas áreas irrigadas cearenses.

A amostra foi constituída por 150 fruticultores, com nível de confiança de $95 \%$ e margem de erro de $8 \%$, sendo distribuída para cada região, conforme a área em hectares destinada ao plantio irrigado de frutas. Com base nas informações da ADECE, Baixo Acaraú, Baixo Jaguaribe, Cariri, Centro-Sul, Ibiapaba e Metropolitana contêm, respectivamente, $2.182 ; 13.145 ; 1.889 ; 926 ; 6.778$ e 4.061 hectares de frutas irrigadas. Assim, foram entrevistados 11 fruticultores do Baixo Acaraú, 68 do Baixo 
Jaguaribe, 10 do Cariri, 5 do Centro-Sul, 35 da região da Ibiapaba e 21 da região Metropolitana.

\section{Resultados e discussões}

\subsection{Caracterização das inovações tecnológicas e de aprendizagem dos fruticultores}

Nessa seção, são mostradas as estatísticas descritivas dos índices de inovação tecnológica e de aprendizagem dos produtores de frutas nas diferentes regiões irrigadas do estado do Ceará. Conforme se observa pela Tabela 1, os fruticultores que se encontram na região Metropolitana apresentam maior índice médio de inovações tecnológicas. Isso pode ser atribuído à proximidade desta região com a capital cearense, onde adquirem máquinas e equipamentos que propiciam melhorias tecnológicas e também possuem acesso a programas de treinamento destinados a novos produtos, assim como a novas formas de comercialização e distribuição desses produtos.

Apesar de a região Metropolitana dispor da maior média do IIT, verificase que as regiões do Baixo Acaraú e do Baixo Jaguaribe contêm fruticultores que detêm o maior valor máximo do IIT da amostra analisada. Esse dado é consistente com a realidade empírica, haja vista que nessas duas regiões se encontram os perímetros públicos federais mais desenvolvidos do Ceará: Tabuleiro de Russas e Jaguaribe-Apodi, no Baixo Jaguaribe, e Baixo Acaraú, localizado na região de mesmo nome. Ademais, há grandes empresas sediadas no Baixo Jaguaribe, como, por exemplo, Agrícola Formosa, Banesa, Del Monte, Fruta Cor e Nolem, cujos produtores investem muito em inovações tecnológicas.

Com relação à dispersão dos produtores de frutas quanto ao IIT, os dados indicam que as regiões do Cariri e do Baixo Acaraú possuem a maior variabilidade, ao passo que na região Centro-Sul, os fruticultores apresentam comportamento tecnológico mais homogêneo. Esse 
comportamento pode ser resultante das diferentes práticas tecnológicas de adubação e pós-colheita adotadas pelos produtores de banana das regiões do Cariri e do Baixo Acaraú, em que os mais tecnificados fazem a adubação via irrigação (fertirrigação), enquanto a adubação é realizada por meio de cobertura para os bananicultores dessas regiões com menor nível tecnológico. Outra diferenciação verificada em tais regiões diz respeito ao uso de tecnologias de pós-colheita, em que os mais tecnificados adotam o processo de climatização para amadurecimento controlado, uma prática não observada para os que detêm menores níveis tecnológicos. No caso da região Centro-Sul, com base na pesquisa de campo, verificouse que todos os produtores de banana entrevistados realizam a adubação nas fases de implantação do plantio e de formação e produção por meio de cobertura e não empregam o processo de climatização para amadurecimento controlado.

Tabela 1 - Estatística descritiva do índice de inovação tecnológica(IIT) dos fruticultores

\begin{tabular}{ccccccc}
\hline \multicolumn{1}{c}{ IIT } & $\begin{array}{c}\text { Baixo } \\
\text { Acaraú }\end{array}$ & $\begin{array}{c}\text { Baixo } \\
\text { Jaguaribe }\end{array}$ & Cariri & $\begin{array}{c}\text { Centro- } \\
\text { Sul }\end{array}$ & Ibiapaba & Metropolitana \\
\hline Valor mínimo & 0,00 & 0,00 & 0,00 & 0,17 & 0,00 & 0,00 \\
Valor médio & 0,26 & 0,31 & 0,23 & 0,30 & 0,25 & 0,40 \\
Valor máximo & 0,83 & 0,83 & 0,67 & 0,50 & 0,67 & 0,67 \\
Desvio padrão & 0,24 & 0,16 & 0,22 & 0,14 & 0,16 & 0,21 \\
$\begin{array}{c}\text { Coeficiente de } \\
\text { variação (\%) }\end{array}$ & 93,15 & 51,14 & 96,42 & 46,47 & 62,79 & 53,91 \\
\hline
\end{tabular}

Fonte: Elaborado pelos autores com base nos resultados da pesquisa.

Os dados da Tabela 2 indicam que os valores médios do índice de aprendizagem foram muito próximos entre as diferentes regiões analisadas, sendo que as regiões do Baixo Jaguaribe e Metropolitana tiveram um valor médio do IA ligeiramente superior, que podem ser justificadas pelas características descritas. Ademais, pode-se inferir que, em termos médios, tanto o IIT quanto o IA apresentaram baixos valores em todas as regiões analisadas. 
Tabela 2 - Estatística descritiva do índice de aprendizagem (IA) dos fruticultores

\begin{tabular}{ccccccc}
\hline IA & $\begin{array}{c}\text { Baixo } \\
\text { Acaraú }\end{array}$ & $\begin{array}{c}\text { Baixo } \\
\text { Jaguaribe }\end{array}$ & Cariri & $\begin{array}{c}\text { Centro- } \\
\text { Sul }\end{array}$ & Ibiapaba & Metropolitana \\
\hline Valor mínimo & 0,17 & 0,10 & 0,20 & 0,20 & 0,10 & 0,10 \\
Valor médio & 0,36 & 0,38 & 0,35 & 0,36 & 0,34 & 0,38 \\
Valor máximo & 0,70 & 0,80 & 0,63 & 0,53 & 0,68 & 0,67 \\
Desvio padrão & 0,16 & 0,13 & 0,14 & 0,12 & 0,14 & 0,16 \\
Coeficiente de & 45,87 & 33,41 & 39,20 & 34,27 & 42,74 & 41,26 \\
variação (\%) & & & & & & \\
\hline
\end{tabular}

Fonte: Elaborado pelos autores com base nos resultados da pesquisa.

Com base nos argumentos apresentados, os produtores de frutas que possuem o maior valor máximo do IA se encontram nas regiões do Baixo Jaguaribe e do Baixo Acaraú. Isso indica que os produtores de frutas que mais investem em inovações tecnológicas também se dedicam ao aprimoramento dos seus conhecimentos na atividade frutícola não apenas via aprendizagem interna e com outros agentes produtivos, mas também por meio de participação em feiras, cursos, eventos e informações de rede colhidas na internet.

Outra evidência que pode ser extraída da Tabela 2 é que os produtores de frutas que apresentam menor heterogenidade quanto ao IA estão no Baixo Jaguaribe, conforme se verifica pelo menor valor do coeficiente de variação. Em contrapartida, a maior variabilidade está presente no Baixo Acaraú.

De posse dessa breve caracterização desses índices analisados neste estudo, é importante conhecer os fatores determinantes que influenciam as escolhas inovativas e de aprendizagem dos fruticultores. Essa questão será apresentada na seção seguinte.

\subsection{Determinantes das inovações tecnológicas e de aprendizagem} dos fruticultores

Para verificar os fatores determinantes das escolhas inovativas e de aprendizagem dos produtores de frutas, os parâmetros foram estimados 
por meio da regressão quantílica para três quantis $(0,25 ; 0,50$ e 0,75$)$, possibilitando captar a influência desses determinantes ao longo de pontos distintos da distribuição condicional dos índices de inovação tecnológica e de aprendizagem e não somente na média.

As estimativas dos modelos que mensuram os efeitos dos determinantes do índice de inovação tecnológica se encontram na Tabela 3. Verifica-se que, entre o grupo de fruticultores que dispõem de menores níveis de inovações tecnológicos, captados pelo quantil 0,25, apenas as dummies Baixo Jaguaribe, Cariri e Centro-Sul não foram significantes. No quantil 0,50 , todos os coeficientes das variáveis explicativas foram significantes a $1 \%$, com exceção das variáveis relativas ao cooperativismo e ao crédito agrícola que não exerceram influência no índice de inovação tecnológica no ponto médio da distribuição. Para o grupo de produtores de frutas que apresentaram melhores níveis tecnológicos, representados pelo quantil 0,75, a única variável incorporada ao modelo que não gerou efeito sobre o IIT foi o crédito agrícola.

O sinal da variável cooperativismo (COOP), quando o coeficiente é significante, foi positivo no quantil 0,25 e negativo no quantil 0,75 , não seguindo, portanto, um padrão homogêneo ao longo da distribuição. 
Tabela 3 - Resultado das estimações: Variável dependente (IIT)

\begin{tabular}{lccc}
\hline \multicolumn{1}{c}{ Variáveis explicativas } & \multicolumn{3}{c}{ Quantis } \\
\cline { 2 - 4 } Constante & 0,25 & 0,50 & 0,75 \\
& 0,0703 & $\mathbf{0 , 1 1 1 2}$ & $\mathbf{0 , 3 2 5 8}$ \\
COOP & $(0,325)$ & $(0,000)$ & $(0,000)$ \\
& $\mathbf{0 , 0 7 0 1}$ & 0,0000 & $\mathbf{- 0 , 0 6 8 2}$ \\
AT & $(0,043)$ & $(1,000)$ & $(0,011)$ \\
& $\mathbf{0 , 0 4 3 8}$ & $\mathbf{0 , 0 5 5 5}$ & $\mathbf{0 , 0 9 0 9}$ \\
ESC & $(0,083)$ & $(0,000)$ & $(0,001)$ \\
& $\mathbf{0 , 0 1 7 5}$ & $\mathbf{0 , 0 2 7 8}$ & $\mathbf{0 , 0 2 2 7}$ \\
CRED & $(0,000)$ & $(0,000)$ & $(0,000)$ \\
Baixo Acaraú & $\mathbf{- 0 , 0 4 3 8}$ & 0,0000 & $-0,0152$ \\
& $(0,092)$ & $(1,000)$ & $(0,465)$ \\
Baixo Jaguaribe & $\mathbf{- 0 , 1 2 2 7}$ & $\mathbf{- 0 , 1 9 4 4}$ & $\mathbf{- 0 , 2 7 2 7}$ \\
Cariri & $(0,025)$ & $(0,000)$ & $(0,000)$ \\
& $-0,0526$ & $\mathbf{- 0 , 0 5 5 5}$ & $\mathbf{- 0 , 0 9 0 9}$ \\
Centro-Sul & $(0,126)$ & $(0,000)$ & $(0,009)$ \\
Ibiapaba & $-0,0966$ & $\mathbf{- 0 , 1 3 8 8}$ & $\mathbf{- 0 , 1 7 4 3}$ \\
& $(0,110)$ & $(0,000)$ & $(0,001)$ \\
& $-0,0439$ & $\mathbf{- 0 , 0 8 3 3}$ & $\mathbf{- 0 , 1 5 1 5}$ \\
\hline
\end{tabular}

Fonte: Elaborado pelos autores com base nos resultados da pesquisa.

Nota: Os valores entre parênteses referem-se ao $p$-value.

O efeito da assistência técnica (AT) foi consistente com o esperado, variando de magnitude ao longo da distribuição. Este resultado corrobora aqueles encontrados por Ribeiro (1989) e Oliveira (2003), que verificaram que a assistência técnica prestada aos produtores ocasiona efeitos positivos sobre a adoção de tecnologia. Portanto, constata-se que os fruticultores assistidos por técnicos possuem maior possibilidade de realizar inovações tecnológicas.

A escolaridade (ESC) também foi uma variável relevante na explicação das diferenças de inovações tecnológicas em todos os quantis estimados. Tal variável apresentou efeito positivo sobre as escolhas inovativas dos fruticultores, de forma que os produtores com maior nível de instrução dispõem de maior nível tecnológico nas três regressões estimadas. Essa relação positiva entre escolaridade e nível tecnológico também foi evidenciada nos estudos de Oliveira (2003) e Matos (2005). 
No caso da variável crédito (CRED), o coeficiente foi significante a $10 \%$ apenas na parte inferior da distribuição da inovação tecnológica, em que a concessão de crédito não é utilizada para a aquisição de inovações tecnológicas. Ademais, essa variável não funciona como um fator relevante para explicar as diferenças observadas no IIT.

Quanto às variáveis dummies regionais, constata-se que os fruticultores localizados nas regiões do Baixo Acaraú e da Ibiapaba apresentaram menor nível de inovação tecnológica se comparados com os sediados na região Metropolitana em todos os quantis analisados. Esse resultado pode estar associado à proximidade entre a região Metropolitana e a capital cearense, facilitando o acesso às inovações tecnológicas.

A Figura 1 permite visualizar os efeitos parciais das variáveis explicativas no índice tecnológico ao longo de toda a distribuição condicional. A área escura indica o intervalo de confiança. Percebe-se, por exemplo, que, apesar de a escolaridade exercer um efeito positivo em toda a distribuição, há, contudo, um efeito positivo com maior intensidade para os quantis superiores da distribuição. Já o efeito da variável Cooperativa muda ao longo da distribuição, apresentando, inclusive, efeito negativo para os quantis intermediários. 

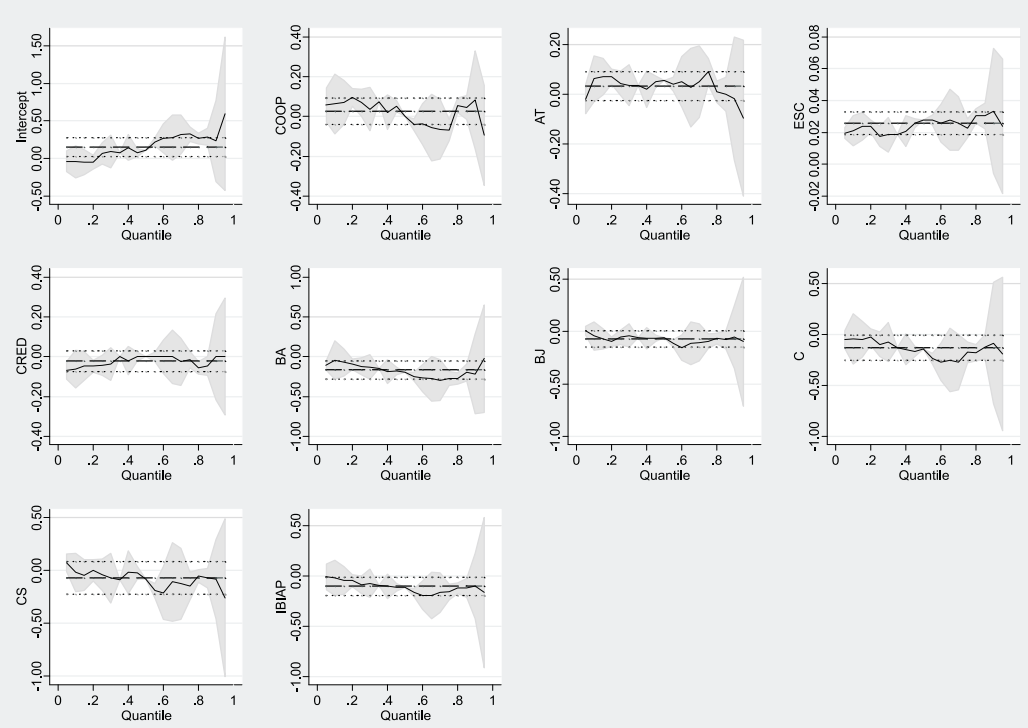

Figura 1 - Resultados das estimações das regressões quantílicas tendo como variável dependente o índice de inovação tecnológica.

Fonte: Elaborado pelos autores com base nos dados da pesquisa.

A Tabela 4 apresenta os resultados das estimativas dos modelos que captam as variáveis que influenciam o índice de aprendizagem. Conforme se observa, entre as variáveis analisadas, a escolaridade é a única que desempenha papel fundamental para explicar variações no nível de aprendizagem em todos os quantis avaliados. O efeito positivo da escolaridade sobre o nível de aprendizagem em toda a distribuição confirma que o grau de instrução do fruticultor está diretamente relacionado com as fontes de informação utilizadas para geração da aprendizagem da atividade frutícola.

O crédito somente é considerado fator determinante para explicar as diferenças entre os níveis de aprendizagem dos produtores com aprendizagem média, porém essa variável apresentou sinal negativo, indicando que o crédito não é empregado pelo produtor para promoção de aprendizagem. 
Tabela 4 - Resultado das estimações: Variável dependente (IA)

\begin{tabular}{lccc}
\hline \multicolumn{1}{c}{ Variáveis explicativas } & \multicolumn{3}{c}{ Quantis } \\
\cline { 2 - 4 } Constante & 0,25 & 0,50 & 0,75 \\
& $\mathbf{0 , 2 1 4 9}$ & $\mathbf{0 , 2 1 6 7}$ & $\mathbf{0 , 1 8 3 3}$ \\
COOP & $(0,000)$ & $(0,000)$ & $(0,006)$ \\
& $-0,0167$ & 0,0000 & 0,0056 \\
AT & $(0,571)$ & $(1,000)$ & $(0,874)$ \\
& 0,0009 & 0,0166 & 0,0519 \\
ESC & $(0,969)$ & $(0,317)$ & $(0,191)$ \\
& $\mathbf{0 , 0 1 4 8}$ & $\mathbf{0 , 0 1 6 7}$ & $\mathbf{0 , 0 2 5 9}$ \\
CRED & $(0,000)$ & $(0,000)$ & $(0,000)$ \\
& $-0,0287$ & $\mathbf{- 0 , 0 3 3 3}$ & 0,0241 \\
Baixo Acaraú & $(0,168)$ & $\mathbf{( 0 , 0 2 1 )}$ & $(0,455)$ \\
& $\mathbf{- 0 , 0 7 8 7}$ & $\mathbf{- 0 , 0 6 6 6}$ & $-0,0204$ \\
Baixo Jaguaribe & $\mathbf{( 0 , 0 7 1 )}$ & $(0,038)$ & $(0,757)$ \\
Cariri & 0,0120 & 0,0333 & 0,0129 \\
& $(0,686)$ & $(0,133)$ & $(0,793)$ \\
Centro-Sul & $-0,0500$ & 0,0166 & 0,0130 \\
Ibiapaba & $(0,327)$ & $(0,616)$ & $(0,854)$ \\
& $-0,0056$ & 0,0000 & $-0,0759$ \\
& $(0,930)$ & $(1,000)$ & $(0,398)$ \\
& $\mathbf{- 0 , 0 6 2 9}$ & 0,0166 & $-0,0112$ \\
& $(0,087)$ & $(0,515)$ & $(0,833)$ \\
\hline
\end{tabular}

Fonte: Elaborado pelos autores com base nos resultados da pesquisa.

Nota: Os valores entre parênteses referem-se ao $p$-value.

Entre as variáveis dummies regionais, verifica-se que o Baixo Acaraú foi significante nos quantis 0,25 e 0,50 , indicando que os produtores dessas regiões até a metade da distribuição registraram menor nível de aprendizagem quando comparados com aqueles que fazem parte da região Metropolitana. A proximidade dessa região com Fortaleza pode contribuir para esse resultado, visto que os fruticultores buscam fontes de informações para o aprendizado da atividade frutícola na capital cearense. Essa evidência também se verifica na região da Ibiapaba na parte inferior da distribuição. As demais variáveis não são fatores relevantes para explicar as diferenças observadas no índice de aprendizagem.

A Figura 2 traz os efeitos das variáveis explicativas ao longo da distribuição condicional do índice de aprendizagem. O efeito da escolaridade no índice de aprendizagem similarmente ao ocorrido no índice tecnológico se dá de forma mais intensa nos quantis superiores só que aqui em maior magnitude. 

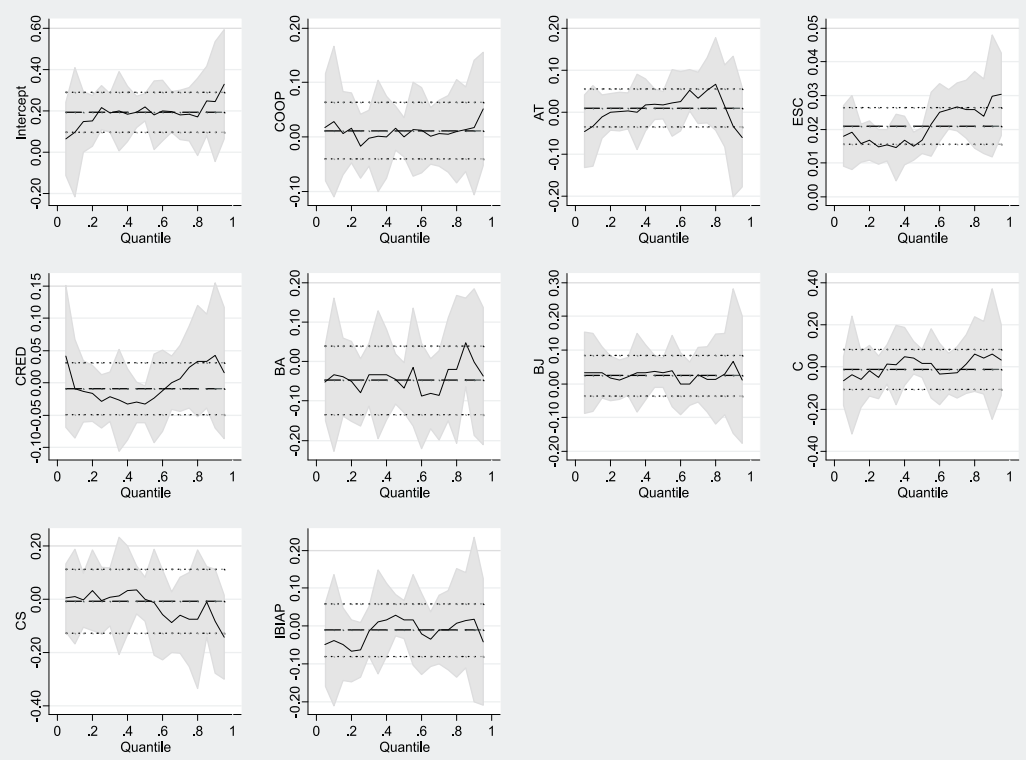

Figura 2 - Resultados das estimações das regressões quantílicas tendo como variável dependente o índice aprendizagem.

Fonte: Elaborado pelos autores com base nos dados da pesquisa.

\section{Conclusões}

Com base nos resultados apresentados neste estudo, constata-se que os fruticultores localizados na região Metropolitana apresentaram maior índice médio de inovações tecnológicas e de aprendizagem, sendo esta região considerada referência para as demais regiões analisadas. Não obstante esse resultado, os produtores de frutas que possuem os maiores valores dos índices de inovações tecnológicas e de aprendizagem se encontram nas regiões do Baixo Jaguaribe e do Baixo Acaraú.

No tocante aos resultados das estimativas das regressões quantílicas, verifica-se que as variáveis escolaridade e assistência técnica exerceram efeitos sobre o índice de inovação tecnológica em todos os quantis 
avaliados. As práticas em atividades cooperativas e o crédito agrícola não foram relevantes para explicar o índice de inovação tecnológica. Ademais, os produtores das demais regiões, notadamente aqueles situados na média da distribuição condicional do índice de inovação tecnológica, apresentaram diferenciais positivos em relação aos produtores localizados na região metropolitana.

Com relação aos determinantes que influenciam o índice de aprendizagem, pode-se inferir que a escolaridade é a única variável que desempenha papel fundamental para explicar variações no nível de aprendizagem ao longo da distribuição. Verificou-se ainda que o fruticultor não utiliza o crédito como forma de obtenção de fontes de informações para promoção de aprendizagem. Os produtores do Baixo Acaraú apresentam diferencial do índice de aprendizagem em relação aos produtores da região metropolitana, especialmente aqueles situados nos quantis inferiores.

\section{Referências}

BANCO DO NORDESTE DO BRASIL. ESPECIALIDADE: Mercado de Frutas e Hortaliças. In: Rede da Irrigação. Documento $\mathrm{n}^{\circ}$. 01. Fortaleza (CE). Disponível em $<\mathrm{http}$ ://www.banconordeste.gov.br/ irriga $>$. Acesso em: 16 de nov. de 2006.

BUCHINSKY, M. Recent advances in quantile regression: a pratical guideline for empirical research. Brown University and NBER, 1997.

DIÁRIO DO NORDESTE. Frutas do Ceará: sabor e oportunidades. Fortaleza, 04 de fevereiro de 2009. 16 p. Disponível em: http:// diariodonordeste.globo.com/caderno.asp?codigo $=181 \&$ CodigoEd. Acesso em: 06/02/2009.

JUSTO, W. R. Políticas sociais e o papel nas disparidades regionais de renda no Brasil: evidências a partir de regressões quantílicas. In: Encontro 
Nacional da Associação Brasileira de Estudos Regionais e Urbanos, 6, 2008. Anais... Aracaju, SE: ENABER, 2008. CDROM.

KOENKER, R.; BASSET, G. Regression quantiles. Econometrica, v. 46, p.33-50, 1978.

MACIEL, M. C.; CAMPÊLO, A. K.; RAPOSO, M. C. F. A dinâmica das mudanças na distribuição salarial e no retorno em educação para mulheres: uma aplicação de regressão quantílica. In: Encontro Nacional de Economia, 29, 2001. Anais... Salvador, BA: ANPEC, 2001.

MATOS, V. D. A apicultura no estado do Ceará: competitividade, nível tecnológico e seus fatores condicionantes, produção e exportação de mel natural. Fortaleza: UFC, 2005. 189 f. Dissertação (Mestrado em Economia Rural) - Universidade Federal do Ceará, 2005.

OLIVEIRA, M. A. S. Nível tecnológico e seus fatores condicionantes na bananicultura do município de Mauriti - CE. Fortaleza: UFC, 2003. 92f. Dissertação (Mestrado em Economia Rural) - Universidade Federal do Ceará, 2003.

RIBEIRO, D. G. L. Adoção de tecnologia na agricultura de canade-açúcar - microrregião do Cariri-CE. Fortaleza: UFC, 1989. 92 f. Dissertação (Mestrado em Economia Rural) - Universidade Federal do Ceará, 1989.

SILVA, S. R.; SILVA, L. M. R.; KHAN, A. S. A fruticultura e o desenvolvimento local: o caso do núcleo produtivo de fruticultura irrigada de Limoeiro do Norte - Ceará. Revista Econômica do Nordeste. Fortaleza, v. 35, n. 1, jan.-mar. 2004.

SOUSA, E. P.; CAMPOS, A. C. Desempenho competitivo dos fruticultores cearenses em diferentes áreas irrigadas. Revista Econômica do Nordeste. Fortaleza, v. 41, n. 1, p. 153-166, 2010. 
SOUZA, P. M.; PONCIANO, N. J. Fatores que interferem nas decisões dos fruticultores da região Norte fluminense. In: Congresso da Sociedade Brasileira de Economia, Administração e Sociologia Rural, 45, 2007. Anais... Londrina, PR: SOBER, 2007. CDROM. 